\title{
USO Y ACTITUDES RELACIONADO A LAS DROGAS EN LAS ESTUDIANTES DE ENFERMERÍA DE LA UNIVERSIDAD MAYOR DE SAN ANDRÉ ${ }^{1}$
}

Iblin Elizabeth Enriquez Flores ${ }^{2}$

Margarita Antonia Villar Luis ${ }^{3}$

Flores IEE, Luis MAV. Uso y actitudes relacionado a las drogas en las estudiantes de enfermería de la Universidad Mayor de San Andrés. Rev Latino-am Enfermagem 2004 março-abril; 12(número especial):376-82.

Este trabajo representa los resultados del Uso y Actitudes relacionando el alcohol, tabaco, Hojas de coca, tranquilizantes y anfetaminas en Estudiantes de Enfermería de la Universidad Mayor de San Andrés. El objetivo es analizar las actitudes mediante el uso de diferentes substancias con aquellos que no usan en los últimos 12 meses. Los datos fueron recolectados en Agosto del 2002, utilizando un cuestionario auto administrado y distribuido, garantizando el anonimato. Se obtuvieron estos resultados: Las actitudes relacionadas a las drogas aprueban el uso de hojas de coca, uso de alcohol, uso de tranquilizantes y desaprueban el uso de cocaína. El uso personal de drogas en los últimos 12 meses corresponde a: hojas de coca diariamente, alcohol menos que una vez por semana y tabaco diariamente. El principal motivo de uso de estas drogas vinculase a la práctica religiosa; los estudiantes son introducidos por primera vez por familiares y por amigos/enamorados.

DESCRIPTORES: uso de drogas; estudiantes universitarios; enfermería

\section{USE AND ATTITUDES ABOUT DRUGS AMONG NURSING STUDENTS AT THE UNIVERSIDAD MAYOR DE SAN ANDRES}

This study presents the results of a research that examined the Use and Attitudes about alcohol, tobacco, coca leaves, tranquilizers and amphetamines among Nursing Students at the Universidad Mayor de San Andrés who had not used these substances over the last 12 months. Data were collected in August 2002 by means of a self-administered and distributed questionnaire, which guaranteed anonymity. The following results were obtained: Attitudes related to drugs approve of coca leaf and tranquilizer use and disapprove of cocaine. Personal use of drugs during the last 12 months corresponds to: daily use of coca leaves, alcohol less than once per week and tobacco every day. The main reason for using these drugs is related to religious practice; the students' first contact with drugs occurs through family members and (girl or boy) friends.

DESCRIPTORS: drugs use; university students; nursing

\section{USO E ATITUDES RELACIONADOS ÀS DROGAS EM ESTUDANTES DE ENFERMAGEM DA UNIVERSIDADE MAIOR DE SAN ANDRÉS}

Este trabalho representa os resultados do Uso e Atitudes relacionados ao álcool, tabaco, folhas de coca, tranqüilizantes e anfetaminas em estudantes de enfermagem da Universidade Mayor de San Andrés. O objetivo é analisar as atitudes mediante o uso das diferentes substâncias com aqueles que usaram ou não drogas nos últimos 12 meses. Os dados da amostra estratificada e intencional foram coletados em agosto de 2002, utilizando um questionário auto administrado distribuído para preservar o anonimato. Obtiveram-se estes resultados: as atitudes relacionadas às drogas demonstram a aprovação do uso das folhas de coca, álcool, tranqüilizantes e desaprovam o uso da cocaína. O uso pessoal de drogas nos últimos 12 meses corresponde a: às folhas de coca diariamente, ao álcool menos que uma vez por semana, e tabaco diariamente. O principal motivo do uso destas drogas vincula-se a prática religiosa; os estudantes são introduzidos pela primeira vez pelos familiares, pelos amigos/enamorados.

DESCRITORES: uso de drogas; estudantes universitários; enfermagem

\footnotetext{
${ }^{1}$ Las opiniones expresadas en este articulo son de responsabilidad exclusiva de los autores y no representan la posición de la organización donde trabajan o de su administración; ${ }^{2}$ Investigadora-licenciada en enfermería, Profesor de la Universidad Mayor de San Andrés, correo electrónico: elyenriquez80@hotmail.com; ${ }^{3}$ Docente de la Escuela de Enfermería de Ribeirão Preto, de la Universidad de São Paulo, Centro Colaborador de la OMS para el desarrollo de la investigación en enfermería, correo electrónico: margarit@eerp.usp.br
} 
INTRODUCCIÓN

El fenómeno de la droga, incluyendo su uso, prevención, dependencia constituye y hace parte de una realidad compleja que tiene que ver con los distintos aspectos de la vida de las personas y la sociedad, entre otros aspectos tiene relación con la salud, educación, cultura, economía, ecología, política, relaciones internacionales, libertad y la autonomía. Continuando con este orden de ideas el abuso de las substancias psicotrópicas rompe el equilibrio biopsicosocial del hombre afectado, dando lugar a importantes alteraciones orgánicas, psicológicas, sociales que afectan a la familia en su integridad psicológica, la armonia de las relaciones interpersonales, altera el presupuesto familiar, se crean situaciones de riesgo en cuanto a la detección de drogas e inducción al uso de otros miembros de la familia. En el ámbito de la sociedad esta practica promueve la corrupción como elemento destructor de la dignidad humana.

En la elaboración de programas de prevención en el uso de drogas para una población específica se debe destacar aspectos importantes tales como: el uso, que se refiere a la cuantificación del consumo, el tipo de drogas usadas, las actitudes o sea el pensamiento, las acciones relacionando las substancias con el conocimiento sobre la acción de las drogas y sus efectos colaterales ${ }^{(1)}$.

La prevalencia del uso de drogas ha sido creciente en todos los lugares del mundo. Los problemas derivados de su uso van desde una criminalidad principalmente al trafico asociado al uso de substancias ilícitas pasando por el uso inadecuado de psicofarmacos que pueden resultar en cuadros de dependencia de substancias licitas y de fácil acceso para el consumo Diferentes referencias bibliográficas especifican y ratifican este comentario ${ }^{(2-3)}$.

La prevalencia en el comportamiento, opinión y actitudes del problema de drogas en Bolivia, corresponde al uso indebido de substancias psicoactivas entre los jóvenes de 12 a 21 años de la población estudiantil los cuales consumen drogas o que al menos han probado y poseen un conocimiento empírico sobre la mezcla y oferta de drogas que existen ${ }^{(4)}$.

Las investigaciones en prevención pasan a buscar una comprensión de como superar la condición de instrumento veiculador de placer que se atribuye a la droga. Muchas son las medidas tentativas para que los jóvenes no se inicien en su uso y para evitar la dependencia. Sin embargo todos los esfuerzos en los últimos años han generado controversias debido a sus enfoques excesivamente intimidatorios aunque estudiosos, profesionales de la salud y autoridades gubernamentales, están de acuerdo en que se trata de un grupo de gran vulnerabilidad proporcionada por un periodo de edad el cual se constituye un riesgo para la salud ${ }^{(5)}$.

\section{OBJETIVO}

Analizar las actitudes relacionadas al uso de drogas buscando diferencias entre los que usan y aquellos que no usan drogas con el propósito de obtener un perfil de la estudiante de la Carrera de Enfermería de la Universidad Mayor de San Andrés durante la gestión 2002.

\section{MATERIAL Y METODOS}

Se utilizó el cuestionario auto-administrado desarrollado en el Brasil por el Grupo Interdisciplinario de Estudios del Alcohol y Drogas en la Facultad de Medicina de la Universidad de São Paulo ${ }^{(6)}$. Se garantizó a los participantes, la confidencialidad y el derecho de no participar o retirarse de la investigación a cualquier momento. Se hicieron algunas adaptaciones necesarias para captar la realidad local (inclusión de la droga, hojas de coca), el cuestionario con 44 variables, lo respondieron 210 universitarios de una muestra intencionada y estratificada. En la variable dependiente "uso de drogas", se consideraron las siguientes drogas: hojas de coca, alcohol, tabaco, cocaína, anfetaminas, tranquilizantes. Las variables independientes fueron agrupadas con las características: personales, del ambiente familiar, ambiente social, vida académica, actitudes, opiniones y uso personal de drogas.

Para facilitar la comprensión de los datos se utiliza el término de uso y no uso de drogas (usuario y no usuario). El patrón escogido es de 12 meses el cual no define el uso frecuente, abuso o dependencia.

\section{RESULTADOS DE LA INVESTIGACIÓN}

\section{Características personales}

A continuación se presenta en tablas conteniendo datos generales sobre los participantes del estudio bien como sobre la práctica del uso de drogas. 
Tabla 1 - Número y porcentaje de estudiantes de enfermería de la UMSA relacionando las características personales con el uso de drogas, 2002

\begin{tabular}{|c|c|c|c|c|c|c|c|}
\hline \multirow[t]{2}{*}{ Variable } & \multirow[t]{2}{*}{ C a tegoría } & \multicolumn{2}{|c|}{$\mathrm{Usa}$} & \multicolumn{2}{|c|}{$\mathrm{NoUsa}$} & \multicolumn{2}{|c|}{ Total } \\
\hline & & $\mathrm{Nr}$ & $\%$ & Nro. & $\%$ & Nro. & $\%$ \\
\hline $\begin{array}{l}\text { Curso } \\
\text { Correspondiente }\end{array}$ & $\begin{array}{l}\text { a) } 1 \text { e r. A ñ o } \\
\text { b) } 2 \text { d o. A ño } \\
\text { c) } 3 \text { e r. A ñ o } \\
\text { d) } 4 \text { to. A ñ o } \\
\text { e) } 5 \text { to. A ñ o }\end{array}$ & $\begin{array}{ll}1 & 6 \\
1 & 8 \\
1 & 1 \\
1 & 4 \\
1 & 1 \\
7 & 0\end{array}$ & $\begin{array}{r}7.62 \\
8.57 \\
5.24 \\
6.66 \\
5.24 \\
33.33\end{array}$ & $\begin{array}{l}32 \\
35 \\
22 \\
27 \\
24 \\
140\end{array}$ & $\begin{array}{l}15.24 \\
16.67 \\
10.48 \\
12.86 \\
11.43 \\
66.67\end{array}$ & $\begin{array}{l}48 \\
55 \\
33 \\
41 \\
35 \\
210\end{array}$ & $\begin{array}{l}22.86 \\
25.24 \\
15.71 \\
19.52 \\
16.67 \\
100\end{array}$ \\
\hline $\begin{array}{l}\text { Sexo } \\
\text { Total }\end{array}$ & $\begin{array}{l}\text { a) Fem enino } \\
\text { b) Masculino }\end{array}$ & $\begin{array}{l}66 \\
4 \\
70\end{array}$ & $\begin{array}{r}31.43 \\
1.90 \\
33.33\end{array}$ & $\begin{array}{l}133 \\
7 \\
140\end{array}$ & $\begin{array}{r}63.33 \\
3.34 \\
66.67\end{array}$ & $\begin{array}{l}199 \\
11 \\
210\end{array}$ & $\begin{array}{c}94.76 \\
5.24 \\
100\end{array}$ \\
\hline $\begin{array}{l}\text { Grupo Etáreo } \\
\text { Total }\end{array}$ & $\begin{array}{l}\text { a) } 18 \text { a } 24 \\
\text { b) } 25 \text { a } 30 \\
\text { c) } 31 \text { y m á s }\end{array}$ & $\begin{array}{l}56 \\
12 \\
2 \\
70\end{array}$ & $\begin{array}{r}26.67 \\
5.71 \\
0.95 \\
33.33\end{array}$ & $\begin{array}{l}111 \\
24 \\
5 \\
\quad 140\end{array}$ & $\begin{array}{r}52.86 \\
11.43 \\
2.39 \\
66.67\end{array}$ & $\begin{array}{l}167 \\
36 \\
7 \\
210\end{array}$ & $\begin{array}{l}79.56 \\
17.14 \\
3.34 \\
100\end{array}$ \\
\hline $\begin{array}{l}\text { Tienes religión } \\
\text { Total }\end{array}$ & $\begin{array}{l}\text { a ) } \mathrm{Si} \\
\text { b) } \mathrm{No}\end{array}$ & $\begin{array}{l}64 \\
6 \\
70\end{array}$ & $\begin{array}{r}30.48 \\
2.85 \\
33.33\end{array}$ & $\begin{array}{l}127 \\
13 \\
140\end{array}$ & $\begin{array}{r}60.48 \\
6.19 \\
66.67\end{array}$ & $\begin{array}{l}191 \\
19 \\
210\end{array}$ & $\begin{array}{c}90.96 \\
9.04 \\
100\end{array}$ \\
\hline $\begin{array}{l}\text { Practicas tu } \\
\text { religión }\end{array}$ & $\begin{array}{l}\text { a) } \mathrm{Si} \\
\text { b) } \mathrm{No}\end{array}$ & $\begin{array}{l}64 \\
6 \\
\end{array}$ & $\begin{array}{r}30.48 \\
2.85 \\
\end{array}$ & $\begin{array}{l}127 \\
13 \\
\end{array}$ & $\begin{array}{r}60.48 \\
6.19 \\
\end{array}$ & $\begin{array}{l}191 \\
19 \\
\end{array}$ & $\begin{array}{r}90.96 \\
9.04 \\
\end{array}$ \\
\hline Total & & 70 & 33.33 & 140 & 66.67 & 210 & 100 \\
\hline
\end{tabular}

Fuente: Dados de la investigación

En relación a las características personales entre la muestra de participantes predomina el sexo femenino, cuyo grupo etáreo más representativo se sitúa entre los 18 a 24 años. Entre los que usan algún tipo de droga, la mayoría de ellos son mujeres y cursando el $2^{\circ}, 1^{\circ}$ y $4^{\circ}$ años, respectivamente. Entre ese grupo $30,4 \%$ tiene y practica religión.

Tabla 2 - Número y porcentaje de estudiantes de enfermería de la UMSA relacionando las características personales con el uso de drogas, 2002

\begin{tabular}{|c|c|c|c|c|c|c|c|}
\hline \multirow[t]{2}{*}{ Variable } & \multirow[t]{2}{*}{ Categoría } & \multicolumn{2}{|c|}{ Usa } & \multicolumn{2}{|c|}{ No Usa } & \multicolumn{2}{|c|}{ Total } \\
\hline & & \multicolumn{2}{|c|}{ Nro. $\%$} & \multicolumn{2}{|c|}{ Nro. $\%$} & \multicolumn{2}{|c|}{ Nro. } \\
\hline \multirow[t]{5}{*}{ Estado Civil } & a) Soltero & 56 & 26.67 & 115 & 54.77 & 171 & 81.44 \\
\hline & b) Casado & 8 & 3.81 & 15 & 7.14 & 23 & 10.95 \\
\hline & c) Divorciado & 2 & 0.95 & 4 & 1.90 & 6 & 2.85 \\
\hline & d) Concubino & 3 & 1.43 & 5 & 2.38 & 8 & 3.80 \\
\hline & e) Viudo & 1 & 0.48 & 1 & 0.48 & 2 & 0.96 \\
\hline Total & & 70 & 33.33 & 140 & 66.67 & 210 & 100 \\
\hline Con quien & a) Padre y Madre & 40 & 19.05 & 81 & 38.57 & 121 & 57.62 \\
\hline \multirow[t]{6}{*}{ Vives } & b) Solamente Madre & 8 & 3.80 & 15 & 7.14 & 23 & 10.94 \\
\hline & c) Solamente Padre & 3 & 1.43 & 5 & 2.38 & 8 & 3.81 \\
\hline & d) Hermanos & 6 & 2.86 & 13 & 6.19 & 19 & 9.05 \\
\hline & e) Esposo e hijos & 9 & 4.29 & 19 & 9.05 & 28 & 13.34 \\
\hline & f) Amigos & 1 & 0.48 & 1 & 0.48 & 2 & 0.96 \\
\hline & g) Otros familiares & 3 & 1.42 & 6 & 2.86 & 9 & 4.28 \\
\hline Total & & 70 & 33.33 & 140 & 66.67 & 210 & 100 \\
\hline Principal & a) Madre & 32 & 15.24 & 62 & 29.53 & 94 & 44.77 \\
\hline \multirow[t]{7}{*}{ Confidente } & b) Padre & 2 & 0.95 & 5 & 2.38 & 7 & 3.33 \\
\hline & c) Hermanos & 9 & 4.28 & 19 & 9.05 & 28 & 13.33 \\
\hline & $\begin{array}{l}\text { d) Amigos } \\
\text { e) Enamorado / }\end{array}$ & 8 & 3.81 & 15 & 7.14 & 23 & 10.95 \\
\hline & Compañero & 7 & 3.34 & 15 & 7.14 & 22 & 10.48 \\
\hline & f) Psicólogo & - & - & - & - & - & - \\
\hline & g) Ninguno & 9 & 4.29 & 18 & 8.57 & 27 & 12.86 \\
\hline & h) Algún Familiar & 3 & 1.42 & 6 & 2.86 & 9 & 4.28 \\
\hline Total & & 70 & 33.33 & 140 & 66.67 & 210 & 100 \\
\hline
\end{tabular}

Fuente: Datos de la investigación 
En la Tabla 2 se observa que $26,6 \%$ de los solteros usan drogas y el $54,7 \%$ no usan, siendo este el dato más representativo de la categoría estado civil. De estos los que viven con ambos padres son el $19,0 \%$ de usuarios y $38,5 \%$, de no usuarios seguido de casados que viven con su esposa e hijos con el $4,2 \%$ y $9,0 \%$ de estos casos, su principal confidente es la madre con el $15,2 \%$ en los que usan y $29,5 \%$ de los que no usan drogas. En esa misma variable se observa la presencia de los hermanos (13,3\%), ningún (12,8\%), amigo (10,9\%) y enamorado o compañero
$(10,4 \%)$, todos estos personajes con puntajes muy próximos. Entre los que hacen uso se observa ese mismo perfil entre las cuatro categorías.

Actitudes de los estudiantes frente al uso de drogas

A continuidad son presentadas las actitudes de los estudiantes en lo referente al uso de alcohol, cocaína, tranquilizantes, anfetaminas y hojas de coca mediante su manifestación a pregunta sobre aprobar o no aprobar que cualquier persona experimente esas substancias.

Tabla 3 - Número y porcentaje de estudiantes de enfermería de la UMSA según uso de drogas y actitudes en cuanto al uso experimental de algunas drogas, 2002

\begin{tabular}{|c|c|c|c|c|c|c|c|}
\hline \multirow{2}{*}{ Variable } & \multirow[t]{2}{*}{ C ategoría } & \multicolumn{2}{|c|}{ Usa } & \multicolumn{2}{|c|}{$\mathrm{NoUsa}$} & \multicolumn{2}{|c|}{ Total } \\
\hline & & & $\%$ & $\mathrm{Nr}$ & $\%$ & $\mathrm{Nrc}$ & $\%$ \\
\hline Experimenta & a) Aprueba & 24 & 11.43 & 49 & 23.33 & 73 & 34.76 \\
\hline Alcohol & b) D es aprueba & 46 & 21.90 & 91 & 43.34 & 137 & 65.24 \\
\hline Total & & 70 & 33.33 & 140 & 66.67 & 210 & 100 \\
\hline $\begin{array}{l}\text { Experim enta } \\
\text { Cocaína } \\
\text { Total }\end{array}$ & $\begin{array}{l}\text { a) A prueba } \\
\text { b) Desaprueba }\end{array}$ & $\begin{array}{l}1 \\
69 \\
70\end{array}$ & $\begin{array}{r}0.48 \\
32.85 \\
33.33\end{array}$ & $\begin{array}{l}1 \\
139 \\
140\end{array}$ & $\begin{array}{r}0.48 \\
66.19 \\
66.67\end{array}$ & $\begin{array}{l}2 \\
156 \\
210\end{array}$ & $\begin{array}{r}0.96 \\
74.29 \\
100\end{array}$ \\
\hline $\begin{array}{l}\text { Experimenta } \\
\text { Tranquilizantes } \\
\text { Total }\end{array}$ & $\begin{array}{l}\text { a) A prueba } \\
\text { b) Desaprueba }\end{array}$ & $\begin{array}{l}18 \\
52 \\
70\end{array}$ & $\begin{array}{r}8.57 \\
24.76 \\
33.33\end{array}$ & $\begin{array}{l}36 \\
104 \\
140\end{array}$ & $\begin{array}{l}17.14 \\
49.53 \\
66.67\end{array}$ & $\begin{array}{l}54 \\
156 \\
210\end{array}$ & $\begin{array}{c}25.71 \\
74.29 \\
100\end{array}$ \\
\hline $\begin{array}{l}\text { Experimenta } \\
\text { Anfetam in as } \\
\text { Total }\end{array}$ & $\begin{array}{l}\text { a) A prueba } \\
\text { b) Desaprueba }\end{array}$ & $\begin{array}{l}9 \\
61 \\
70\end{array}$ & $\begin{array}{c}4.28 \\
29.05 \\
33.33\end{array}$ & $\begin{array}{l}16 \\
124 \\
140\end{array}$ & $\begin{array}{r}7.62 \\
59.05 \\
66.67\end{array}$ & $\begin{array}{l}25 \\
185 \\
210\end{array}$ & $\begin{array}{r}11.90 \\
88.10 \\
100\end{array}$ \\
\hline $\begin{array}{l}\text { Experimenta } \\
\text { Hojas de coca }\end{array}$ & $\begin{array}{l}\text { a) A prueba } \\
\text { b) Desaprueba }\end{array}$ & $7^{63}$ & $\begin{array}{l}30 \\
3.33 \\
\end{array}$ & $\begin{array}{l}127 \\
13 \\
\end{array}$ & $\begin{array}{r}60.48 \\
6.19 \\
\end{array}$ & $\begin{array}{l}190 \\
20 \\
\end{array}$ & $\begin{array}{r}90.48 \\
9.52 \\
\end{array}$ \\
\hline Total & & 70 & 33.33 & 140 & 66.67 & 210 & 100 \\
\hline
\end{tabular}

Fuente: Datos de la investigación

Los datos indican que el mayor índice de desaprobación entre el grupo en general recae sobre las anfetaminas, seguido de los tranquilizantes y cocaína y por fin el alcohol, aunque todos están muy próximos en porcentaje. Sin embargo, la experimentación de hojas de coca tiene el mayor porcentaje de aprobación $(90,4 \%)$. Entre los estudiantes que hacen uso los mayores índices de desaprobación se refieren al uso de la cocaína, y de las anfetaminas, ya los tranquilizantes y el alcohol se presentan con puntajes muy próximas.

Tabla 4 - Número y porcentaje de estudiantes de enfermería de la UMSA según uso de drogas y actitudes en cuanto al uso regular de algunas drogas, 2002

\begin{tabular}{|c|c|c|c|c|c|c|c|}
\hline \multirow[t]{2}{*}{ Variable } & \multirow[t]{2}{*}{ C a tegoría } & \multicolumn{2}{|c|}{ U s a } & \multicolumn{2}{|c|}{$\mathrm{No} \cup \mathrm{sa}$} & \multicolumn{2}{|c|}{ Total } \\
\hline & & $\mathrm{N}$ & $\%$ & $\mathrm{~N} \mathrm{r}$ & $\%$ & $\mathrm{Nr}$ & $\%$ \\
\hline $\begin{array}{l}\text { Uso regular } \\
\text { De alcohol } \\
\text { Total }\end{array}$ & $\begin{array}{l}\text { a) A pruebo } \\
\text { b) D esapruebo }\end{array}$ & $\begin{array}{ll}1 & 6 \\
5 & 4 \\
7 & 0\end{array}$ & $\begin{array}{l}7.62 \\
25.71 \\
33.33\end{array}$ & $\begin{array}{lll}3 & 3 \\
1 & 07 \\
1 & 4 & 0\end{array}$ & 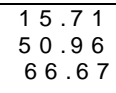 & $\begin{array}{l}49 \\
161 \\
210\end{array}$ & $\begin{array}{l}23.33 \\
76.67 \\
100\end{array}$ \\
\hline $\begin{array}{l}\text { Uso regular } \\
\text { De cocaína } \\
\text { Total }\end{array}$ & $\begin{array}{l}\text { a) A pruebo } \\
\text { b) Desapruebo }\end{array}$ & $\begin{array}{l}1 \\
69 \\
70\end{array}$ & $\begin{array}{l}\quad 0.47 \\
32.86 \\
33.33\end{array}$ & $\begin{array}{l}1 \\
139 \\
140\end{array}$ & $\begin{array}{c}0.48 \\
66.19 \\
66.67\end{array}$ & $\begin{array}{lll}2 & & \\
2 & 0 & 8 \\
2 & 1 & 0\end{array}$ & $\begin{array}{r}0.95 \\
99.05 \\
100\end{array}$ \\
\hline $\begin{array}{l}\text { Uso regular de } \\
\text { tranquilizantes } \\
\text { Total }\end{array}$ & $\begin{array}{l}\text { a) A pruebo } \\
\text { b) D esapruebo }\end{array}$ & $\begin{array}{l}13 \\
57 \\
70\end{array}$ & $\begin{array}{r}6.19 \\
27.14 \\
33.33\end{array}$ & $\begin{array}{ll}2 & 5 \\
1 & 15 \\
140\end{array}$ & $\begin{array}{llll}1 & 1 & .9 & 1 \\
5 & 4.7 & 6 \\
6 & 6.6 & 7\end{array}$ & $\begin{array}{l}38 \\
172 \\
210\end{array}$ & $\begin{array}{llll}1 & 8.1 & 0 \\
8 & 1.9 & 0 \\
1 & 0 & 0\end{array}$ \\
\hline $\begin{array}{l}\text { Uso regular de } \\
\text { anfetam inas } \\
\text { Total }\end{array}$ & $\begin{array}{l}\text { a) A pruebo } \\
\text { b) D esapruebo }\end{array}$ & $\begin{array}{l}6 \\
64 \\
70\end{array}$ & $\begin{array}{r}2.86 \\
30.47 \\
33.33\end{array}$ & $\begin{array}{l}13 \\
127 \\
140\end{array}$ & $\begin{array}{r}6.19 \\
60.48 \\
66.67\end{array}$ & $\begin{array}{l}19 \\
191 \\
210\end{array}$ & $\begin{array}{r}9.05 \\
90.95 \\
100\end{array}$ \\
\hline $\begin{array}{l}\text { Uso regular de } \\
\text { Hojas de coca }\end{array}$ & $\begin{array}{l}\text { a) A pruebo } \\
\text { b) Desapruebo }\end{array}$ & $\begin{array}{l}66 \\
4 \\
\end{array}$ & $\begin{array}{r}31.43 \\
1.90 \\
\end{array}$ & $\begin{array}{l}132 \\
8\end{array}$ & $\begin{array}{c}62.86 \\
3.81 \\
\end{array}$ & $\begin{array}{l}198 \\
12\end{array}$ & $\begin{array}{r}94.29 \\
5.71 \\
\end{array}$ \\
\hline Total & & 70 & 33.33 & 140 & 66.67 & 210 & 100 \\
\hline
\end{tabular}

Fuente: Datos de la investigación 
En la Tabla 4 se verifica que el uso regular ocurre en mayor porcentaje con los usuarios de las hojas de coca con $31,4 \%$, aprobando su uso; seguido por usuarios que aprueban el uso del alcohol con 7,6\%; continuando por usuarios que aprueban el uso de tranquilizantes con un $6,1 \%$, correspondiendo las menores cifras a los usuarios que aprueban el uso de anfetaminas con el $2,8 \%$ y los usuarios que aprueban el uso de la cocaína con el 0,4\% según muestran esos datos la tolerancia para el uso regular de hojas de coca predomina, de largo, sobre las demás substancias.

Tabla 5 - Número y porcentaje de estudiantes de enfermería de la UMSA según uso de drogas e información relacionadas al uso personal de drogas, 2002

\begin{tabular}{|c|c|c|c|c|c|c|c|}
\hline \multirow[t]{2}{*}{ Variable } & \multirow[t]{2}{*}{ C ategoría } & \multicolumn{2}{|c|}{ Usa } & \multirow{2}{*}{\multicolumn{2}{|c|}{ No Usa }} & \multicolumn{2}{|c|}{ Total } \\
\hline & & & $\%$ & $\mathrm{NrC}$ & & Nro & $\%$ \\
\hline $\begin{array}{l}\text { Uso alcohol últim os } \\
12 \text { m eses } \\
\text { Total }\end{array}$ & $\begin{array}{l}\text { a) } \mathrm{Si} \\
\text { b) } \mathrm{No}\end{array}$ & $\begin{array}{l}23 \\
47 \\
70\end{array}$ & $\begin{array}{l}10.96 \\
22.38 \\
33.33\end{array}$ & $\begin{array}{l}46 \\
94 \\
140\end{array}$ & $\begin{array}{l}21.90 \\
44.76 \\
66.67\end{array}$ & $\begin{array}{l}69 \\
141 \\
210\end{array}$ & $\begin{array}{c}32.86 \\
67.14 \\
100\end{array}$ \\
\hline $\begin{array}{l}\text { Uso tabaco últimos } \\
12 \text { meses } \\
\text { Total }\end{array}$ & $\begin{array}{l}\text { a) } \mathrm{Si} \\
\text { b) } \mathrm{No}\end{array}$ & $\begin{array}{l}15 \\
55 \\
70\end{array}$ & $\begin{array}{r}7.14 \\
26.19 \\
33.33\end{array}$ & $\begin{array}{l}31 \\
109 \\
140\end{array}$ & $\begin{array}{l}14.76 \\
51.91 \\
66.67\end{array}$ & $\begin{array}{l}46 \\
164 \\
210\end{array}$ & $\begin{array}{c}21.90 \\
78.10 \\
100\end{array}$ \\
\hline $\begin{array}{l}\text { Uso cocaína últimos } \\
12 \text { meses } \\
\text { Total }\end{array}$ & $\begin{array}{l}\text { a) } \mathrm{Si} \\
\text { b) } \mathrm{No}\end{array}$ & $\begin{array}{l}1 \\
69 \\
70\end{array}$ & $\begin{array}{rl} & 0.48 \\
32 & 2.85 \\
3 & 3.33\end{array}$ & $\begin{array}{l}2 \\
138 \\
140\end{array}$ & $\begin{array}{r}0.95 \\
65.72 \\
66.67\end{array}$ & $\begin{array}{l}3 \\
207 \\
210\end{array}$ & $\begin{array}{r}1.43 \\
98.57 \\
100\end{array}$ \\
\hline $\begin{array}{l}\text { Uso anfetam inas } \\
\text { últimos } 12 \mathrm{meses} \\
\text { Total }\end{array}$ & $\begin{array}{l}\text { a) } \mathrm{Si} \\
\text { b) } \mathrm{No}\end{array}$ & $\begin{array}{c}9 \\
61 \\
70\end{array}$ & $\begin{array}{l}4.29 \\
29.05 \\
33.33\end{array}$ & $\begin{array}{l}19 \\
121 \\
140\end{array}$ & $\begin{array}{r}9.05 \\
57.62 \\
66.67\end{array}$ & $\begin{array}{l}28 \\
182 \\
210\end{array}$ & $\begin{array}{c}13.34 \\
86.67 \\
100\end{array}$ \\
\hline $\begin{array}{l}\text { Uso tranquilizantes } \\
\text { últimos } 12 \mathrm{meses} \\
\text { Total }\end{array}$ & $\begin{array}{l}\text { a) } \mathrm{Si} \\
\text { b) } \mathrm{No}\end{array}$ & $\begin{array}{l}16 \\
54 \\
70\end{array}$ & $\begin{aligned} & 7.62 \\
& 25.71 \\
& 33.33\end{aligned}$ & $\begin{array}{l}31 \\
109 \\
140\end{array}$ & $\begin{array}{l}14.76 \\
51.90 \\
66.67\end{array}$ & $\begin{array}{l}47 \\
163 \\
210\end{array}$ & $\begin{array}{c}22.38 \\
77.62 \\
100\end{array}$ \\
\hline $\begin{array}{l}\text { Uso Hojas de coca } \\
\text { últimos } 12 \mathrm{meses}\end{array}$ & $\begin{array}{l}\text { a) } \mathrm{Si} \\
\text { b) } \mathrm{No}\end{array}$ & $\begin{array}{l}64 \\
6 \\
\end{array}$ & $\begin{array}{r}30.48 \\
2.85 \\
\end{array}$ & $\begin{array}{l}128 \\
12 \\
\end{array}$ & $\begin{array}{r}60.95 \\
5.72 \\
\end{array}$ & $\begin{array}{l}192 \\
18 \\
\end{array}$ & $\begin{array}{r}91.43 \\
8.57 \\
\end{array}$ \\
\hline
\end{tabular}

Fuente: Datos de la investigación

En cuanto al uso personal de drogas en los últimos 12 meses la Tabla 5, muestra un porcentaje de uso alto, entre los estudiantes, de hojas de coca con $91,4 \%$, luego en seguida, el uso de alcohol con $32,8 \%$, continuando el uso de tranquilizantes con el $22,3 \%$, el tabaco con $21,9 \%$, las anfetaminas con $13,3 \%$ y por último la cocaína con $1,4 \%$.
Informaciones sobre el uso personal de drogas

La Tabla 6 muestra los motivos para usar por primera vez drogas, quienes las introdujeron en el uso y si esto ocurrió antes o después de su ingreso en la universidad.

Tabla 6 - Número y porcentaje de estudiantes de enfermería de la UMSA relacionando información con el uso personal de drogas, 2002

\begin{tabular}{|c|c|c|c|c|c|c|c|}
\hline \multirow[t]{2}{*}{ Variable } & \multirow[t]{2}{*}{ C a tegoría } & \multicolumn{2}{|c|}{ Us a } & \multicolumn{2}{|c|}{$\mathrm{NoU}$ s a } & \multicolumn{2}{|c|}{ Total } \\
\hline & & \multicolumn{2}{|c|}{ N ro } & \multicolumn{2}{|c|}{ N ro } & \multirow{2}{*}{$\mathrm{Nrc}$} & $\%$ \\
\hline Principal motivo & a) $\mathrm{No}$ uso & 29 & 13.80 & 59 & 28.10 & & 28.10 \\
\hline porprimera vez & b) Practica religios a & 10 & 4.76 & 19 & 9.04 & 29 & 13.80 \\
\hline de drogas & $\begin{array}{l}\text { c) Porrelación } \\
\text { d) Diversión o placer } \\
\text { e) Am igos/Enamorado }\end{array}$ & $\begin{array}{l}6 \\
6\end{array}$ & $\begin{array}{l}2.86 \\
2.86\end{array}$ & $\begin{array}{ll}13 \\
11\end{array}$ & $\begin{array}{l}6.19 \\
5.24\end{array}$ & $\begin{array}{l}19 \\
17\end{array}$ & $\begin{array}{l}9.05 \\
8.10\end{array}$ \\
\hline & us a n & 7 & 3.33 & 15 & 7.15 & 22 & 10.48 \\
\hline & f) Curiosidad & 6 & 2.86 & 12 & 5.71 & 18 & 8.57 \\
\hline & g) Estrés & 3 & 1.43 & 7 & 3.33 & 10 & 4.76 \\
\hline & h) A u m entar desempeño & 2 & 0.95 & 3 & 1.43 & 5 & 2.38 \\
\hline & i) Otros & 1 & 0.48 & 1 & 0.48 & 2 & 0.96 \\
\hline Total & & 70 & 33.33 & 140 & 66.67 & 210 & 100 \\
\hline Introducción por & a) $\mathrm{No}$ uso & 33 & 15.71 & 66 & 31.43 & 99 & 47.14 \\
\hline primera vez de & b) F a m iliares & 12 & 5.71 & 26 & 12.38 & 38 & 18.10 \\
\hline drog a s & $\begin{array}{l}\text { c) Am igos } \\
\text { d) Enamorado/ }\end{array}$ & 11 & 5.24 & 23 & 10.95 & 34 & 16.19 \\
\hline & Com pañ e ro & 7 & 3.33 & 12 & 5.71 & 19 & 9.05 \\
\hline & e) Otros & 7 & 3.33 & 13 & 6.19 & 20 & 9.52 \\
\hline Total & & 70 & 33.33 & 140 & 66.67 & 210 & 100 \\
\hline Experimento drogas & & & & & & & \\
\hline antes de su ingreso & a) $\mathrm{S} \mathrm{i}$ & 31 & 14.76 & 61 & 29.05 & 92 & 43.81 \\
\hline a la Universidad & b) $\mathrm{No}$ & 39 & 18.57 & 79 & 37.62 & 118 & 56.19 \\
\hline Total & & 70 & 33.33 & 140 & 66.67 & 210 & 100 \\
\hline
\end{tabular}

Fuente: Datos de la investigación 
Según se observa, el principal motivo para los estudiantes introducirse por primera vez en las drogas es la practica religiosa con un $11,9 \%$. La introducción por primera vez a las drogas en los estudiantes ocurre entre los familiares con $18,1 \%$. Se debe subrayar que la porcentaje de alumnos que experimentaron drogas después de su ingreso en la universidad está bastante próxima de aquella de los que lo hicieron antes (18,57\% y $14,76 \%$, respectivamente). Los amigos/enamorados, también aparecen ser una influencia importante, tanto en la motivación para el uso, como en la experimentación de las drogas.

\section{DISCUSIÓN}

Esta investigación parte de un proyecto con el propósito de comprender las actitudes relacionadas al uso de las drogas en jóvenes universitarios con la visión de elaborar campañas de prevención. Las medidas de uso (cantidad y frecuencia), la definición de droga, substancias investigadas son las principales diferencias en los estudiantes universitarios. Así como la especificación de la muestra, tipo de análisis y el formato usado para análisis de datos, aunque el cuestionario haya mostrado efectividad en la obtención de informaciones, para realizar una comparación metodológica con estudios nacionales, existe una limitante por la escasez de estudios de esta naturaleza, así como de investigaciones volcadas a analizar las actitudes de estudiantes universitarios relacionados al uso de drogas $^{(7-8)}$.

El uso de medicamentos en países menos avanzados como Bolivia con legislaciones débiles o que no se cumplen, existe un tolerado y franco abuso en la dispensación de medicamentos, incluso la venta sin necesidad de receta médica, destinados a remediar todas las tensiones y ansiedades de la vida ${ }^{(9)}$. Las hojas de coca utilizadas por la población Boliviana debido a las creencias de los beneficios que ofrece tales como: ser anoréxico; en la altura a nivel del mar tiene cierto efecto broncodilatador, previne la caries dental cuando mascada $^{(4)}$.

Los resultados de esta investigación muestran que predomina el sexo femenino pues esta es una característica de la profesión de enfermería. Son jóvenes que viven con sus padres, mismo aquellas que han hecho uso de alguna droga, dato este que no se muestra de acuerdo con el estudio de Queiroz ${ }^{(6)}$.
También hay diferencias cuanto al motivo principal por el cual han hecho uso experimental de drogas, esto es, el religioso. Puede ser que tal diferencia sea derivada de razones culturales, ya que la droga más utilizada en los últimos 12 meses son las hojas de coca y inclusive estas también poseen el más bajo índice de desaprobación ${ }^{(10)}$. Se observa aún que el uso por primera vez ha dado en casa o con amigos/enamorados. Este resultado si concuerda con otros estudios ${ }^{(3,11)}$.

Las actitudes de la muestra relacionadas a las drogas esta representadas en mayores cifras por la aprobación del uso de alcohol, hojas de coca, tranquilizantes. Las comparaciones del uso legal e ilegal refieren tanto a la facilidad de su obtención como aceptación de la sociedad. La desaprobación de la cocaína inclusive el uso experimental rompe la hipótesis de que Bolivia de productores de hojas de coca pasaría a consumidor.

La muestra estudiantil del presente estudio está compuesto por universitarios que han vencido los cursos pre facultativos y en su generalidad provienen de escuelas públicas y su ingreso ha requerido una selección en su proceso educacional para posibilitar su ingreso a la universidad, estos estudiantes corresponderían al grupo de no usuarios de drogas con el $67 \%$. Sin embargo un grupo considerable de ellos ya ha tenido contacto con drogas antes del ingreso en la universidad por tanto el entorno de sus actividades y sus relaciones sociales podrían constituirse factores de riesgo en el uso de drogas. Es imprescindible realizar estrategias de prevención e información relacionado al uso de substancias psicoactivas, por el acceso fácil a las drogas tanto lícitas como ilícitas así como la fuerte influencia cultural respecto al uso de las hojas de coca.

\section{AGRADECIMIENTOS}

Agradecemos a todos los docentes de la Escuela de Enfermería de Ribeirão Preto y a los treinta y tres participantes de nueve países latinoamericanos que participaron en el proceso de implementación de los programas, y también al Gobierno del Japón y al Programa de Becas de la OEA por su apoyo financiero y becas que posibilitaron la implementación del "I Programa Regional de Capacitación en Investigación para Enfermeros de América Latina". 


\section{REFERENCIAS BIBLIOGRÁFICAS}

1. Nicastre S, Ramos PS. Prevenção do uso de drogas. Gaz Bras Dep Quim 2001; 2 (Suplemento 1):25-9.

2. Galduroz JCF, Noto AR, Carlini EA. IV Levantamento sobre o uso de drogas entre estudantes de 1 e $2^{\circ}$ graus em 10 capitais brasileiras. São Paulo: CEBRID Centro Brasileiro de Informações sobre drogas psicotropicas. Departamento de Psicologia, Escola Paulista de Medicina, Universidade Federal Paulista; 1997.

3. Carlini ELA. Uso ilícito de drogas lícitas pela nossa juventude. É um problema solúvel: Rev Bras Crescimento Desenvol Humano 1992; 2(1):129-43.

4. Alcazar Del CF. El uso indebido de drogas em estudiantes de Bolivia. Bolivia: Ed. Grafico; 1999.

5. Leukefeld CG, Bukoski WJ. An Introduction to drug abuse, prevention, research: methodological sigues. Rock Ville: Nida; 1999, p. 1-6.

6. Queiroz S. Fatores relacionados ao uso de drogas e condições de risco entre alunos de graduação da USP. [dissertação]. São Paulo (SP): Faculdade de Saúde Pública/ USP; 2000.

7. Prendergast ML. Substance use and abuse among college students: a review of recent lite rature. J Addic Coll Health 1994 Nov; 43:99-113.

8. Andrade AG, Bassit AZ, Mesquita AM, Fukushima JT, Gonçalves EL. Prevalência do uso de drogas entre alunos da faculdade de medicina da Universidade de São Paulo - 19911993. Revista ABP-APAL 1995; 17(2):41-6.

9. Acción Internacional por lá Salud. Trabajando por una mejor salud! Ahora! 2001; 13.

10. Cronin C. Religiosity, religious affiliation, and alcohol and drugs use among American students living in Germany. Int J Addic 1995 Jan; 30(2):231-8.

11. Micheli D, Formigoni ML. As razões para o primeiro uso de drogas e as circunstâncias familiares prevêen os padrões de uso futuro? Gaz Bras Dep Quim 2001; 2(1):20-30. 